\title{
Ankylosing hyperostosis
}

\section{Clinical and radiological features}

\author{
JACQUELINE HARRIS, A. R. CARTER, E. N. GLICK, AND G. O. STOREY \\ From the Department of Rheumatology, Hackney Hospital, and the Department of Radiology, The London \\ Hospital, London
}

Ankylosing hyperostosis is a condition in which new bone is laid down on the right antero-lateral aspect of the dorsal vertebrae and across the intervertebral spaces forming spurs or bridges. The lumbar and cervical spines, pelvis, hips, and knees may also be involved. There is no single approved name for this condition. Oppenheimer (1942) described 'Calcification and ossification of vertebral ligaments'. Forestier and Rotes-Quérol (1950) named it 'senile ankylosing hyperostosis of the spine', but in later works (Forestier and Lagier, 1971) the adjective 'senile' was omitted when the condition was described in younger patients. Smith, Pugh, and Polley (1955) described 'physiologic vertebral ligamentous calcification' and Hájková, Strěda, and Škrha (1965) 'hyperostotic spondylosis'. Beardwell (1969) termed this condition 'ankylosing vertebral hyperostosis' and Julkunen, Heinonen, and Pyörälä (1971) referred to 'hyperostosis of the spine'. Bywaters and Forestier (1967) presented a revised system for nomenclature of spinal diseases at the 6th European Congress of Rheumatology. They advised that this condition be named 'ankylosing hyperostosis', and this is the terminology which we have adopted.

An association with diabetes mellitus has been shown by several authors, including Boulet and Mirouze, (1954), Hájková and others (1965), Bywaters, Doyle, and Oakley (1966), and Ott, Perkovac, and Regehr (1967). Julkunen and others (1971) found an association with diabetes and obesity. Boulet, Serre, and Mirouze (1954) suggested that this condition is due to an increased production of growth hormone occurring when the secretion of gonadal hormones declines, and in fact, Julkunen, Kärävä, and Viljanen (1966) found six cases of hyperostosis of the spine in 21 acromegalic patients. Beardwell (1969) showed a familial incidence and relationship with tylosis in a Greek Cypriot family. Ott (1953) and Smith and others (1955) considered the disease to be an exaggerated form of osteoarthrosis of the spine.

Although many European authors have described the clinical, radiological, and pathological features of ankylosing hyperostosis, there has been no report of a series of patients with this condition from this country. This paper describes the clinical and radiological features of a series of patients seen at various hospitals in the East End of London. Particular reference has been made to the possible association with acromegaly.

\section{Material and methods}

For inclusion in this survey, the following radiological criteria had to be fulfilled: hypertrophic spurs in the dorsal spine predominantly on the right side with at least two bony bridges; absence of other spinal disease which might cause bony bridging (e.g. collapsed vertebra or gross scoliosis); absence of sacroiliitis. The study was purposely limited to patients with definite and severe involvement. All patients were questioned about symptoms referable to the spine and peripheral joints. The reason for attending hospital, trauma to back, occupation, racial origin, and history of diabetes mellitus in the patient or his family were also recorded. Spinal movements were graded as normal, slightly restricted, severely restricted, or nil. The height and weight of each patient were measured, and any clinical evidence of acromegaly, tylosis, or psoriasis was noted.

Antero-posterior and lateral radiographs were taken of the entire spine, and radiographs of the pelvis, hands, knees, and skull were also obtained in nearly all patients. All observed abnormalities in the spinal films were noted with particular reference to the presence of bony spurs or bridges, their locations (i.e. right, left, anterior, or a combination) and to the presence of new bone on the anterior surfaces of the vertebral bodies. When present, intervertebral disc narrowing with sclerosis of the adjacent vertebral plates, apophyseal joint involvement, and osteoporosis were also recorded. The name 'bony spur' was used for a projection from a vertebral body which extended in a vertical direction either up or down beyond the end-plate. The term 'bridge' was used when there appeared to be bony union between two such spurs. The term 'osteophyte' was reserved for a bony projection from the margin of a vertebral body which tended to project laterally and did not extend vertically beyond the endplate of the vertebral body. In practice, this differentiation was usually possible, but there were a few examples which were difficult to classify, particularly in the cervical region. 
In difficult cases, bony projections in the anterior and posterior directions which were associated with disc space narrowing were termed osteophytes. The films of the hands, knees, and pelvis were assessed for evidence of osteoarthrosis and the skull and hands for acromegalic changes.

The following laboratory investigations were performed: haemoglobin, white cell count, erythrocyte sedimentation rate, latex fixation, serum calcium, phosphorus, alkaline phosphatase, and spot blood sugar. The urine was tested for glucose. In five of the younger patients serum growth hormone levels were estimated by the method of Hartog, Gaafar, Meisser, and Fraser (1964).

\section{Results}

\section{(l) Clinical}

34 patients were studied: seventeen men aged 47 to 82 years (mean 68) and seventeen women aged 47 to 83 years (mean 66). Only eleven came from the British Isles. Nineteen were Jewish-usually from Russia or Poland-two West Indian, one Cypriot, and one Italian. This racial distribution probably reflects the number of immigrants living in this area rather than a racial predominance.

No patient gave a history of spinal trauma and none had done heavy manual work. Five patients had no backache but the rest had pain in some region of the back.

\section{DORSAL SPINE}

Thirteen patients had had some pain in the dorsal spine; of these, two had experienced pain many years previously and six had only mild intermittent discomfort. The remaining five complained of more definite pain which was variously described as constant, worse on coughing, worse on lifting, brief, nocturnal, and to the right of the spine. The duration of symptoms ranged from 2 to 28 years. Five complained of stiffness of the dorsal spine. There was no relationship between symptoms and the extent of dorsal spine involvement as seen radiologically. Two patients derived some relief from indomethacin or phenylbutazone.

Rotation of the dorsal spine was tested with the patient sitting with arms folded. 28 had some limitation of movement, but none had a completely rigid spine. There was a mild kyphosis in seven patients.

\section{LUMBAR SPINE}

23 patients had pain in the lumbar spine; the description was usually vague but was not usually that of a disc lesion. Five complained of stiffness. Movements were usually limited, but the lumbar spine was never rigid although forward flexion and extension were limited in 25 patients and lateral flexion in 29. Nineteen patients with symptoms referable to the lumbar spine had radiological changes of hyperostosis in this region.

\section{CERVICAL SPINE}

Eleven patients complained of severe pain in the cervical spine whilst five experienced mild discomfort. All had radiological abnormalities-seven hyperostosis, six cervical spondylosis, and three a combination of the two conditions.

\section{PER I PHERAL JOINTS}

24 patients complained of pain in the peripheral joints: shoulders (10), knees (8), hips (5), feet and ankles (5), hands (4). Two patients had more generalized pain characteristic of polymyalgia rheumatica. Twenty patients were suffering from various pathological conditions: ischaemic heart disease (4), bronchitis (2), gout (1), chondrocalcinosis (6), thyrotoxicosis (1), gallstones (1), hiatus hernia (1), duodenal ulcer (1), old poliomyelitis (1), Paget's disease (2).

There were four diabetics (duration 1 to 20 years), but only one required insulin therapy. Four others had a family history of diabetes mellitus in siblings or mother. None of the non-diabetics had glycosuria and all had spot blood sugars of less than $180 \mathrm{mg} . / 100 \mathrm{ml}$. Glucose-tolerance tests were performed in six patients and all were normal.

Using tables for average weights of adults (Documenta Geigy, 1970), 30 patients were overweight. Thirteen had Heberden's nodes. None appeared clinically acromegalic, and none had psoriasis or tylosis.

One patient was anaemic with a haemoglobin of $10.8 \mathrm{~g}$. per cent. and an erythrocyte sedimentation rate of $60 \mathrm{~mm} . / 1 \mathrm{st} \mathrm{hr}$. No cause has been found for these abnormalities. The blood count and sedimentation rate were normal in all other patients at the time of survey. The latex-fixation test was negative in all cases and calcium and phosphorus estimations were normal in the 30 patients who had these investigations performed. The main laboratory test to show any abnormality was the alkaline phosphatase level which was mildly raised in seven cases.

Growth hormone levels were estimated in five of the younger patients (three women aged 67, 59, and 56 years and two men aged 47 and 58 years). None was diabetic. All had normal growth hormone levels, i.e. fasting up to $60 \mu \mathrm{g}$./litre suppressing to less than $10 \mu \mathrm{g}$./litre in response to glucose.

\section{(2) Radiological}

The most characteristic pattern of bony spurs and bridges was seen in the dorsal spine, where they predominated on the right and anterior aspects of the vertebrae, particularly in the lower dorsal region with the maximum number being situated at the D8/9 level (Figs 1, 2 and 3). They were also present on the left side, but always less than on the right. The deposition of new bone on the anterior surfaces of the 
dorsal vertebral bodies correlated closely with the levels involved by spurs and bridges and in fact the new bone usually merged with the latter. Generally, the changes seen in the cervical and lumbar regions were less marked and did not seem to have such a distinctive pattern of distribution.

Radiological abnormalities of the cervical spine were present in 31 patients. Twelve were considered to have cervical spondylosis alone, viz. disc space narrowing and anterior and posterior osteophytes; thirteen had hypertrophic spurs or bridges with preservation of the disc spaces; six had a mixture of the

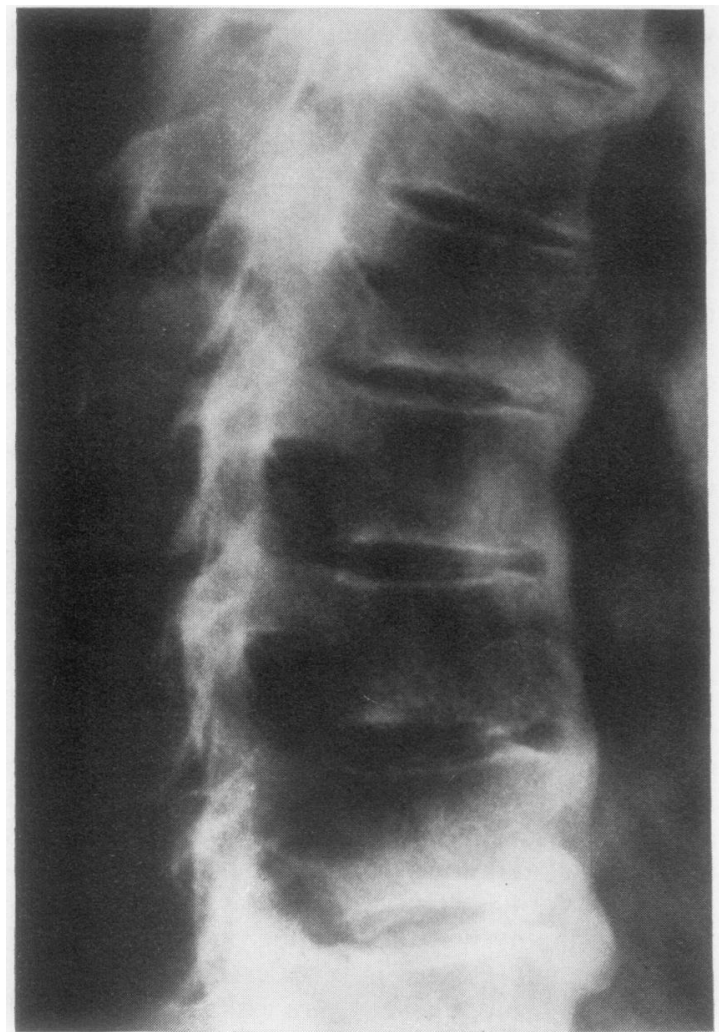

FIG. 1 Lateral radiograph of lower dorsal spine, showing multiple spurs and bridges anteriorly

two types. In the group with hypertrophic changes alone, four had particularly striking changes with bridging between the majority of the vertebral bodies and considerable new bone formation anteriorly (Fig. 4). Discrete areas of well-corticated bone in the mid-line posterior to the spinous processes of $\mathrm{C} 4,5$, and 6 were seen in three patients.

Bony bridging between vertebrae and new anterior bone at two or more levels in the lumbar region were unusual, being seen in four and eight patients respectively. Spurs, however, were seen in 27 patients (Fig. 5).

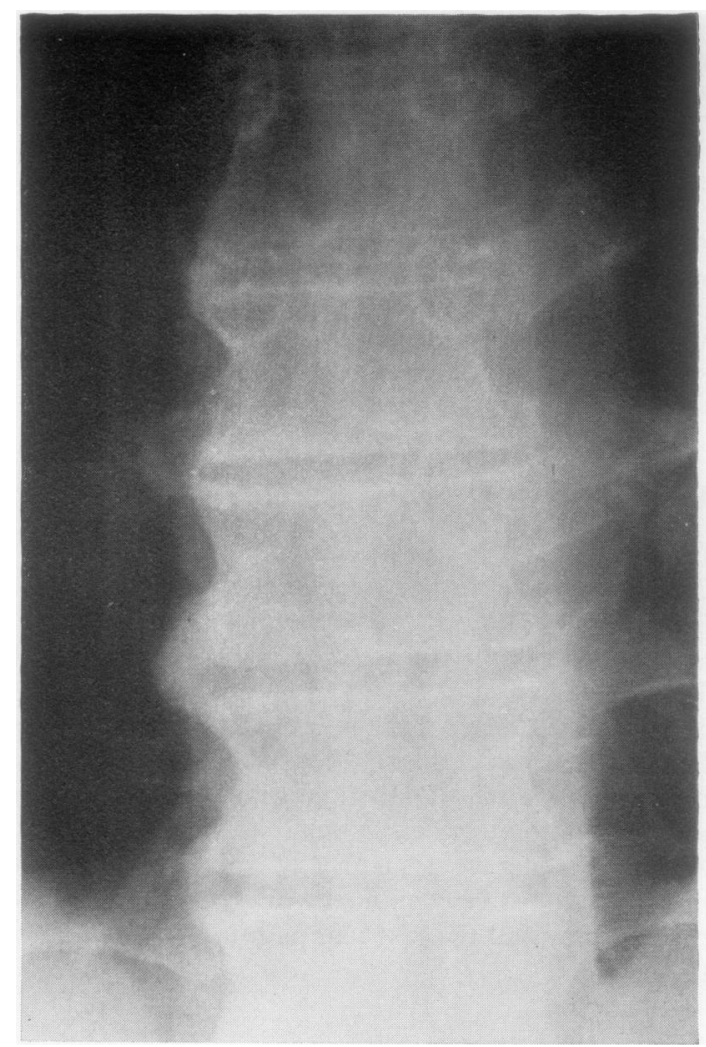

FIG. 2 Antero-posterior radiograph of lower dorsal spine, showing two bridges on the right side and lack of involvement on the left

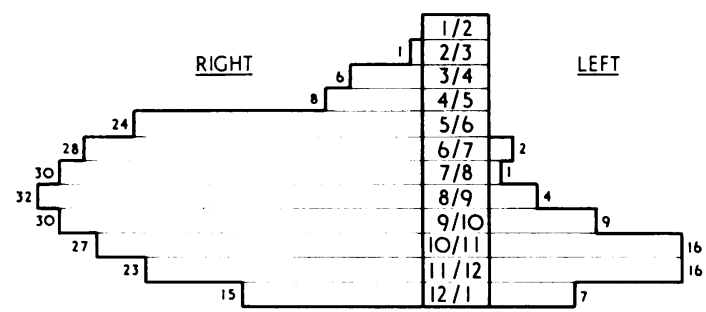

FIG. 3 Levels of dorsal spine involvement with spurs or bridges in 34 patients

Eighteen of the 30 patients ( 60 per cent.) who had pelvic radiographs had 'fluffy' new bone formation, typically on the margins of the iliac bones from the anterior superior iliac spines to just above the acetabulae (Fig. 6), inferiorly in relation to the pubic rami, and in relation to the femoral greater trochanters.

In two patients, similar changes were related to the femoral and tibial condyles and one had new bone formation around both fibular heads.

No patient was considered to have sacroiliitis. Two had spurs at the lower ends of the sacroiliac joints 


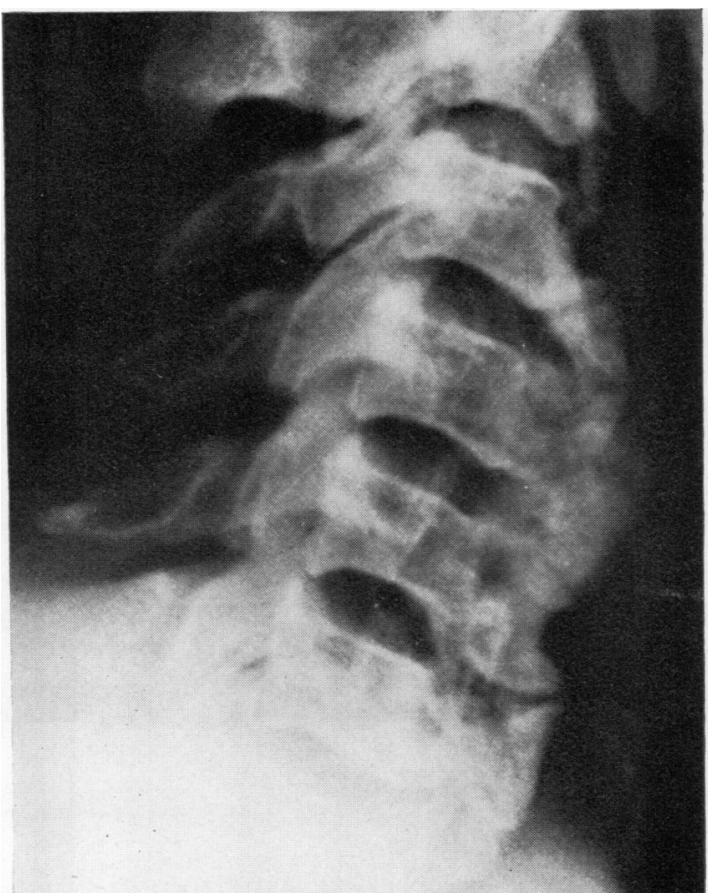

FIG. 4 Lateral radiograph of lower cervical spine, showing very extensive new bone formation anteriorly with bridging at multiple levels. The disc spcices are maintained

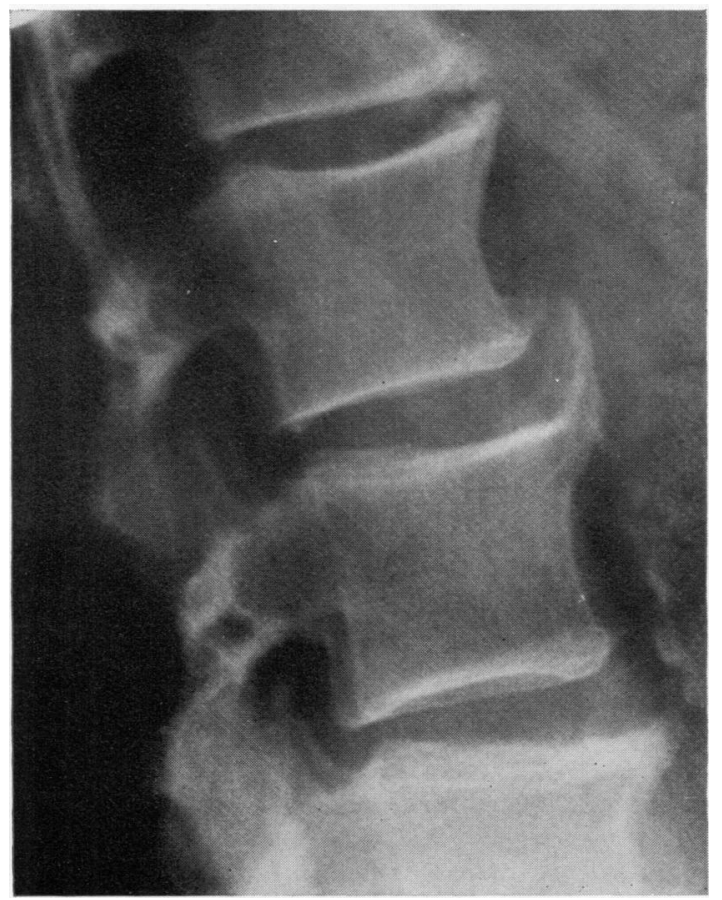

FIG. 5 Lateral radiograph of upper lumbar spine. Spurs at several levels but no bridges (there is calcification in the abdominal aorta, anterior to the body of L3)

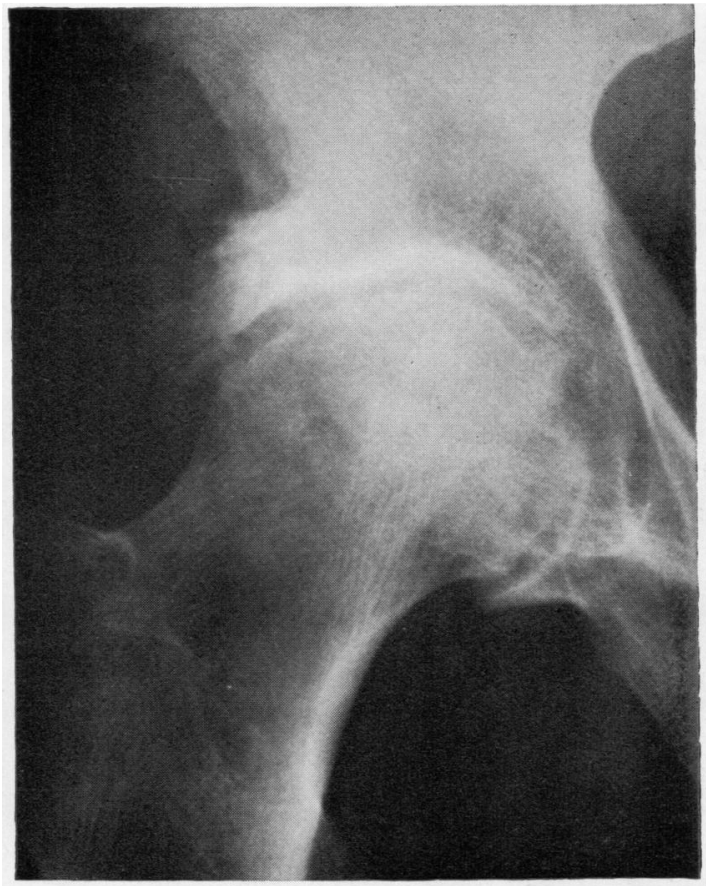

FIG. 6 Antero-posterior radiograph of hip joint. There is 'fluffy' periostitis adjacent to the acetabulum superiorly

similar to those seen between vertebral bodies. One had osteitis condensans ilii and another had sclerosis of uncertain cause on the iliac side of the left sacroiliac joint superiorly.

The following additional findings were noted:

Chondrocalcinosis in knees or hips (six patients).

Paget's disease in pelvis and/or lumbar vertebrae (two patients).

Hypoplasia of the left side of the pelvis associated with old poliomyelitis (one patient).

Changes of osteoarthrosis were extremely common in the hands and knees, being seen in 28 ( 90 per cent.) of the 31 patients examined. However, these changes were always minimal.

Narrowing of the L4/5 disc space associated with a grade I spondylolisthesis (one patient).

Narrowing of the L2/3 and L3/4 disc spaces in relation to a minor scoliosis (one patient).

Hyperostosis frontalis interna of the skull with no evidence of diabetes mellitus or acromegaly (one patient).

Enlarged pituitary fossa without any confirmatory radiological evidence of acromegaly (one patient); this patient on further investigation showed no evidence of pituitary abnormality.

Radiological assessment of osteoporosis on plain films is notoriously difficult, but no patient had any 
evidence of vertebral collapse or striking loss of bone density.

\section{Discussion}

In making a radiological diagnosis of ankylosing hyperostosis, other causes of spinal calcification and ossification must be excluded. The typical appearance of late ankylosing spondylitis is well known and usually presents no diagnostic difficulties. Riley, Ansell, and Bywaters (1971) have suggested, however, that when this condition presents in older patients the typical syndesmophyte of ankylosing spondylitis may become distorted and look like an osteophyte or hypertrophic spur. This is possibly due to an associated bulging of the related disc. The presence of sacroiliitis should serve to make the distinction between the two conditions. Paraspinal ossification has been described in four patients with psoriasis (Bywaters and Dixon 1965) - but the new bone in such cases is separate from the vertebral bodies and is situated mainly on the lateral aspects. Reiter's syndrome may be associated with bony bridging of the vertebrae-Cliff (1971) described four cases with 'skip' lesions in the spine. In hypoparathyroidism there may be extensive calcification of the posterior paraspinal ligaments and apophyseal articulations with fused osteophytes and increased bone density in the spine (Jimenea, Frame, Chaykin, and Sigler, 1971). Fluorosis is another cause of calcification of intervertebral ligaments and lipping of the vertebral bodies, but the generalized increase in bone density is usually distinctive (Siddiqui, 1955). In all these conditions, the clinical picture and laboratory tests will help to establish the diagnosis.

It must be emphasized that ankylosing hyperostosis cannot be diagnosed with certainty merely by looking at the radiograph of an isolated area of the spine. The large hypertrophic spurs are different from the classical syndesmophytes and osteophytes as described by Riley and others (1971), but we saw some examples which were indistinguishable. Murray, Oates, and Young (1958) emphasized that 'there is a limited number of ways in which a bone or joint can react to a pathogenic agent; it is clear that different aetiological factors may cause identical radiographic changes'.

The present study has confirmed the observations of previous workers (Forestier and Lagier, 1971) that the bony bridges predominate on the right side of the dorsal region and that spurs are more common than bridges in the cervical and lumbar regions. Forestier and Lagier suggested that the relative mobility of the cervical and lumbar regions spared them from bridge formation and that the pulsation of the descending aorta served to protect the left side of the dorsal region from spur and bridge formation. These explanations seem plausible but it would be in- teresting to see if the pattern was different in a patient with a right-sided aorta.

'Fluffy' new bone formation related to the pelvis, femora, and tibiae was seen with sufficient frequency to suggest that it is a feature of this condition. In fact, the diagnosis was often made from a pelvic radiograph and then confirmed by $x$-ray examination of the dorsal spine. Similar distinctive changes were sometimes seen around other joints.

Islands of bone lying posterior to the lower cervical spinous processes in the nuchal ligament seen in three patients are also seen in patients with cervical spondylosis and patients with normal cervical spines. It is probably not a feature of ankylosing hyperostosis and may be related to previous trauma.

The general lack of disc space narrowing and the absence of apophyseal joint involvement in the dorsal and lumbar spines was particularly striking in this group of elderly patients; although 90 per cent. had radiological evidence of osteoarthrosis of the hands or knees, this was usually minimal. Only three had osteoarthritic hips. From this, it would seem unlikely that this condition is a manifestation of generalized osteoarthrosis. However, thirteen patients had Heberden's nodes.

The hypothesis that ankylosing hyperostosis is a form of acromegaly has not been substantiated by this study. No patient had the clinical features of acromegaly and five had normal growth hormone levels. Although one had an enlarged pituitary fossa on the lateral skull radiograph, no abnormality of pituitary function could be shown in this patient, and none had any other definite radiological features of the condition on skull or hand radiographs. It should be emphasized, however, that radiographs of the spine may not always be sufficient to distinguish between these two conditions with certainty. Bluestone, Bywaters, Hartog, Holt, and Hyde (1971) described the clinical and radiological features of acromegalic arthropathy in 42 patients. Findings in the dorsal spine included kyphosis, osteophytes, bridges, bone deposition on the anterior vertebral surfaces, and scalloping of the vertebral bodies posteriorly. An increase in the height of the disc spaces was especially marked in the lumbar region. In the later stages of the disease, they considered the radiological appearances to be very similar to those seen in ankylosing hyperostosis. It is noteworthy that clinically their patients demonstrated increased spinal mobility, and it was suggested that this excess mobility was the initiating factor for osteophyte formation.

Symptoms in this series were very variable. Five had no pain but most had some backache and this was sometimes severe. Some limitation of spinal movement was usually found, but the spine was not rigid and this served to distinguish the condition clinically from ankylosing spondylitis. 


\section{Summary}

The clinical and radiological features of 34 patients with ankylosing hyperostosis are presented.

Pain in some region of the back was found in 29 cases.

The condition may sometimes be suspected clinically by the presence of moderate to severe limitation of back movements (without rigidity) in an obese elderly patient, but the diagnosis can usually only be made radiologically.
The peripheral joints and the pelvis may be affected by fluffy periostitis.

No abnormality in growth hormone levels was found and the hypothesis that this condition is a form of acromegaly was not substantiated.

We wish to thank Dr. Barbara Boucher for the growth hormone analysis, and Dr. G. B. Laird, Dr. D. T. D. Hughes, and Dr. A. Freedman for allowing us to study their patients.

\section{References}

Beardwell, A. (1969) Ann. rheum. Dis., 28, 518 (Familial ankylosing vertebral hyperostosis with tylosis)

Bluestone, R., Bywaters, E. G. L., Hartog, M., Holt, P. J. L., AND Hyde, S. (1971) Ibid., 30, 243 (Acromegalic arthropathy)

Boulet, P., ANd Mirouze, J. (1954) Ann. Méd., 55, 674 (Les ostéoses diabétique (ostéoporose et hyperostose))

- Serre, H., and Mirouze, J. (1954) Sem. Hôp. Paris, 30, 2392 (Le rachis diabétique)

Bywaters, E. G. L., AND Dixon, A. St. J. (1965) Ann. rheum. Dis., 24, 313 (Paravertebral ossification in psoriatic arthritis)

—, Doyle, F. H., AND OAKLEY, N. (1966) Arthr. and Rheum., 9, 495 (Senile hyperostotic ankylosing spondylosis in diabetes mellitus)

- AND ForestiER, J. (1967) 'Reports and Abstracts of VI European Congress of Rheumatology', p. 472 (Illustrated Glossary of Terminology)

Cliff, J. M. (1971) Ann. rheum. Dis., 30, 171 (Spinal bony bridging and carditis in Reiter's disease)

Documenta Geigy (1970) 'Scientific Tables', 7th ed., ed. K. Diem and C. Lentner, p. 711 (Average weights of adults). Geigy, Basel, Switzerland

FoRESTIER, J., AND LAGIER, R. (1971) Clin. orthop., 74, 65 (Ankylosing hyperostosis of the spine)

- AND ROTES-QuÉROL, J. (1950) Ann. rheum. Dis., 9, 321 (Senile ankylosing hyperostosis of the spine)

HÁJKová, Z., STřEdA, A., AND ŠKRHA, F. (1965) Ibid., 24, 536 (Hyperostotic spondylosis and diabetes mellitus)

Hartog, M., GaAfar, M. A., Meisser, B., AND Fraser, R. (1964) Brit. med. J., 2, 1229 (Immunoassay of serum growth hormone in acromegalic patients)

Jimenea, C. V., Frame, B., Chaykin, L. B., ANd Sigler, J. W. (1971) Clin. orthop., 74, 84 (Spondylitis of hypoparathyroidism)

Julkunen, H., Heinonen, O. P., ANd Pyörala, K. (1971) Ann. rheum. Dis., 30, 605 (Hyperostosis of the spine in an adult population. Its relation to hyperglycaemia and obesity)

- KÄRÄVÄ, R., AND VILJANEN, V. (1966) Diabetologia (Berl.), 2, 123 (Hyperostosis of the spine in diabetes mellitus and acromegaly)

Murray, R. S., OAtes, J. K., AND Young, A. C. (1958) J. Fac. Radiol., 9, 37 (Radiological changes in Reiter's syndrome)

OPPENHEIMER, A. (1942) Radiology, 38, 160 (Calcification and ossification of vertebral ligaments (spondylitis ossificans ligamentosa): roentgen study of pathogenesis and clinical significance)

OTT, V. R. (1953) Schweiz med. Wschr., 83, 790 (Über die Spondylosis hyperostotica)

-, Perkovac, N., AND Regehr, I. (1967) 'Reports and Abstracts of VI European Congress of Rheumatology', p. 517 (Hyperostotic spondylosis and ankylosing spondylitis. Correlation with disorders of carbohydrate metabolism)

Riley, M. J., Ansell, B. M., AND Bywaters, E. G. L. (1971) Ann. rheum. Dis., 30, 138 (Radiological manifestations of ankylosing spondylitis according to age at onset)

SidDiQuI, A. H. (1955) Brit. med. J., 2, 1408 (Fluorosis in Nalgonda District, Hyderabad-Deccan)

Smith, C. F., Pugh, D. G., ANd Polley, H. F. (1955) Amer. J. Roentgenol., 74, 1049 (Physiologic vertebral ligamentous calcification: an aging process) 